\title{
Surgical management of retrorectal tumors: a retrospective study of a 9-year experience in a single institution
}

This article was published in the following Dove Press journal:

OncoTargets and Therapy

29 November 20II

Number of times this article has been viewed

\author{
Caizhao Lin' \\ Ketao Jin ${ }^{2,3}$ \\ Huanrong $\operatorname{Lan}^{4}$ \\ Lisong Teng ${ }^{2}$ \\ Jianjiang Lin' \\ Wenbin Chen' \\ 'Department of Coloproctological \\ Surgery, First Affiliated Hospital, \\ College of Medicine, Zhejiang \\ University, Hangzhou, ${ }^{2}$ Department \\ of Surgical Oncology, First Affiliated \\ Hospital, College of Medicine, \\ Zhejiang University, Hangzhou, \\ ${ }^{3}$ Department of Surgery, Affiliated \\ Zhuji Hospital, Wenzhou Medical \\ College, Zhuji, ${ }^{4}$ Department of \\ Gynecology and Obstetrics, Affiliated \\ Taizhou Hospital, Wenzhou Medical \\ College, Linhai, Zhejiang, People's \\ Republic of China
}

Background: The relative rarity and anatomical position of retrorectal tumors may lead to difficulty in diagnosis and surgical management.

Methods: This was a retrospective review of 62 patients who had resection of retrorectal tumors between 2002 and 2010.

Results: All patients in this study were treated by excision of the retrorectal tumors. Surgical approach included transsacral approach (52 cases), transabdominal approach (eight cases), and combined approach (two cases). A total of 48 benign lesions (77.4\%) and 14 malignant lesions (22.6\%) were confirmed by histological examination. The 48 benign cases included dermoid cysts (17 cases), simple cysts (eight cases), teratomas (eight cases), neurofibromas (eight cases), fibrolipomas (four cases), neurilemmomas (two cases), and synovioma (one case). The 14 malignant cases included lymphomas (four cases), malignant teratomas (three cases), fibrosarcomas (two cases), interstitialomas (four cases) and malignant mesothelioma (one case). Complications occurred in $14.5 \%$ of patients and included intraoperative bleeding (three cases), rectal injury (three cases), and presacral infection (three cases).

Conclusion: Primary retrorectal tumors are very rare. Successful treatment of these tumors requires extensive knowledge of pelvic anatomy and expertise in pelvic surgery.

Keywords: retrorectal space, retrorectal tumor, surgical treatment

\section{Background}

Tumors within the retrorectal space are uncommon and due to their rarity and diverse symptoms they are often misdiagnosed or mistreated. Tumors occurring in the retrorectal space are extremely rare in the adult population, with an estimated incidence of 0.0025-0.014 in large referral centers. ${ }^{1}$ Retrorectal tumors may be classified as congenital, neurogenic, osseous, or miscellaneous. ${ }^{2}$ Two-thirds are congenital, caused by embryological sequestration, abnormalities in midline fusion, and incomplete embryological regression. Cystic congenital lesions include epidermoid and dermoid cysts, tailgut cyst (also called cystic hamartoma or mucus-secreting cyst), enterogenic cyst, teratoma, and teratocarcinoma. ${ }^{2,3}$ Neurogenic (including anterior sacral meningoceles), osseous, and miscellaneous tumors each account for approximately $10 \%$ of retrorectal tumors. ${ }^{2,3}$ Retrorectal tumors remain a difficult diagnostic and therapeutic challenge despite the use of newer imaging modalities and improvements in perioperative care. The diagnosis of these lesions requires a high index of suspicion, and treatment should be undertaken only by surgeons with expertise in complex pelvic operations. ${ }^{2}$ Most retrorectal tumors ultimately require surgical resection. Complete resection with negative margins is the standard for benign retrorectal tumors, but it
Correspondence: Lisong Teng First Affiliated Hospital, College of Medicine, Zhejiang University, 79, Qingchun Road, Hangzhou, Zhejiang 310003 ,

People's Republic of China

Tel $+8657 \mid 87068873$

Fax +8657187236628

Email jinketao200I@zju.edu.cn 
is more difficult to resect malignant tumors to bring about a cure, and the tumors tend to recur locally. The aim of this study was to review the authors' surgical experience with retrorectal tumors over the past 9 years.

\section{Methods}

The clinical records of a series of 62 patients who underwent surgery for retrorectal tumors from 2002 to 2010 at the Department of Coloproctological Surgery, First Affiliated Hospital, College of Medicine, Zhejiang University, were reviewed. Patient demographics, symptoms, physical findings, investigations, surgical interventions, pathology, and complications were all documented. Follow-up information was supplemented by means of a telephone interview with each patient. The study received ethics board approval at First Affiliated Hospital.

\section{Results}

A total of 62 patients underwent surgery for a retrorectal tumor, of which 23 were men and 39 women. Ages ranged from 15 to 68 years (median 40.5 ), with $67.7 \%$ of them aged $<40$ years (42 cases). The clinical course ranged from 1 week to 10 years (median 10.2 months). Forty-five patients were preliminarily diagnosed and 17 were follow-up patients. The diameter of the tumors ranged from 3 to $15 \mathrm{~cm}$. Twenty-four patients $(38.7 \%)$ were asymptomatic and the retrorectal mass were discovered on routine screening rectal examination. In the remaining $38(62.3 \%)$ patients, 22 complained of pain in lumbosacral area and eight in the hypogastric area, while eight had a palpable mass on physical examination. Seven patients $(14.5 \%)$ had difficulty defecating, four $(8.3 \%)$ had lower extremity pain, three $(6.25 \%)$ had lower extremity edema, two $(4.1 \%)$ had dysuria, and two (4.1\%) had perineal fistulae.

Forty patients underwent routine ultrasonography as the only diagnostic evaluation. Of the 28 patients diagnosed, twelve had solid or solid cystic tumors. Eight of the 28 patients with cystic tumors underwent transrectal ultrasound during their initial evaluation for a retrorectal mass, all being reported presacral tumors. Fifteen patients received pelvic plain radiographs. Eight of them showed calcification in the presacral area, three had compressioninduced sacral and vertebral deformity, and one showed pelvic wall involvement with bony destruction, while the remaining three patients did not show any positive signs. Twenty patients underwent computed tomography scans to confirm the diagnosis (Figure 1). The border of the presacral mass was clear in twelve and unclear in eight of them. Pelvic magnetic resonance imaging (MRI) was performed on twelve

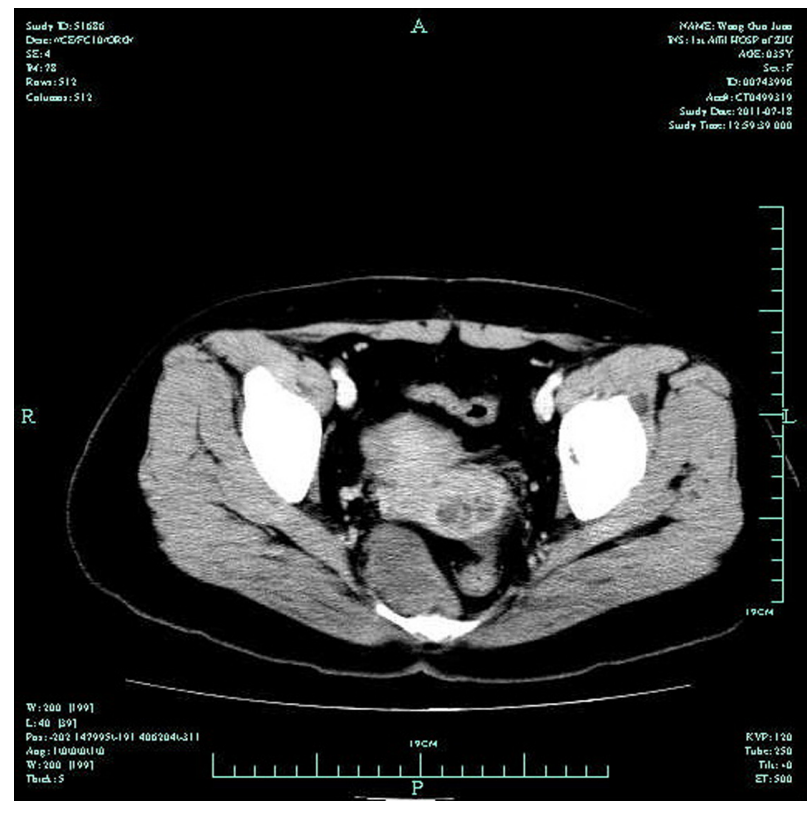

Figure I Computed tomographic image of neurolemmoma.

suspected cases (Figure 2A and B), in which ten of the masses were in the presacral area and two were of rectal origin. Other imaging modalities such as contrast perineum fistulography were used in two cases.

All patients in this study were treated with excision of the retrorectal tumors. The surgical approach included transsacral approach in 48 cases, transabdominal approach in eight cases, and combined abdominosacral approach in six cases. Four of the 52 cases which underwent transsacral approach were intraoperatively converted to a combined approach. The excisions were microscopically complete in 52 cases. With an additional six palliative resections, the resection rate was $93.5 \%$. One teratocarcinoma with rectum involvement received a combined resection of tumor and rectum followed by a coloproctostomy. Three lymphomas with extensive rectal, sacrococcygeal, pelvic, and mesenteric lymph node metastasis had simple sigmoid colostomies.

Complications occurred in $14.5 \%$ of patients. There were three intraoperative bleedings (of more than $800 \mathrm{~mL}$ ), including one benign and two malignant cases. There were also three rectal injuries, one receiving simple repair of the rectum and the other two receiving partial rectal resection with rectal anastomosis. Presacral infection occurred in three cases after operation. Two of these were cured after sufficient drainage and the other received an additional epluchage after 1 month of drainage, which was considered successful.

A total of 48 benign (77.4\%) and 14 malignant (22.6\%) lesions were confirmed by histological examination (Table 1). The 48 benign cases included 17 dermoid cysts, 


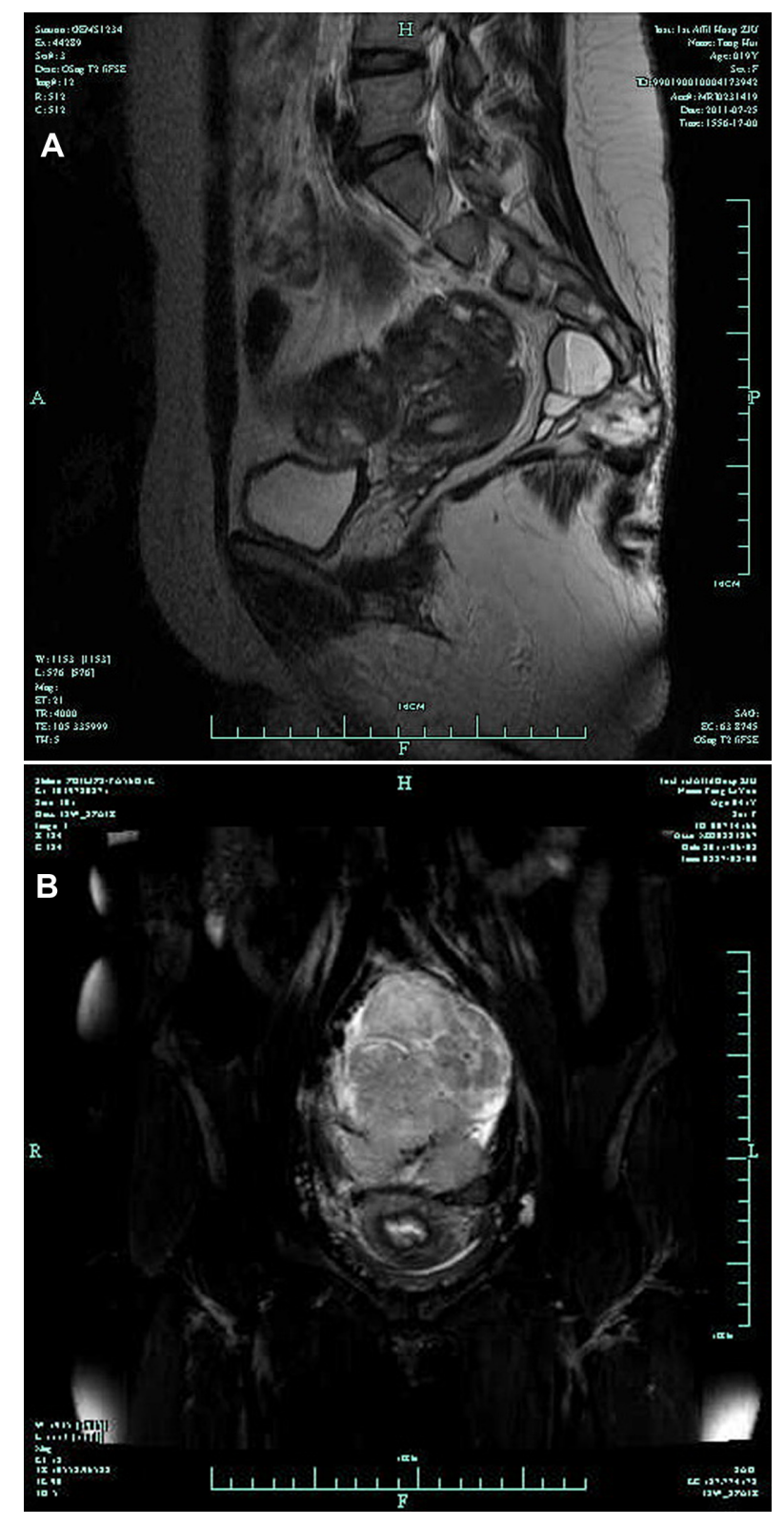

Figure 2 Magnetic resonance imaging images of dermoid cyst (A) and fibrosarcoma (B).

eight simple cysts, eight teratomas, eight neurofibromas, four fibrolipomas, two neurilemmomas and one synovioma. The 14 malignancy included four lymphomas, three malignant teratomas, two fibrosarcomas, four interstitialomas, and one malignant mesothelioma. Forty-five patients were followed-up for 6 months to 9 years, and seven of them experienced at least one episode of recurrence, including two neurofibromas and one local recurrence of interstitialoma.

\section{Discussion}

Primary tumors of the retrorectal (or presacral) space are rare lesions. Retrorectal tumors produce symptoms that are often
Table I Histopathological findings and clinical outcome

\begin{tabular}{lll}
\hline Histopathology & $\mathbf{n}(\%)$ & Recurrence \\
\hline Benign & $48(77.4)$ & \\
Dermoid cyst & I $7(27.4)$ & None \\
Simple cyst & $8(I 2.9)$ & None \\
Teratoma & $8(I 2.9)$ & I (I episode) \\
Neurofibroma & $8(I 2.9)$ & 2 (I episode) \\
Fibrolipoma & $4(6.5)$ & I (I episode) \\
Neurilemmoma & $2(3.2)$ & None \\
Synovioma & I (I.6) & None \\
Malignant & I $4(22.6)$ & \\
Lymphoma & $4(6.5)$ & I (local) \\
Interstitialoma & $4(6.5)$ & I (local) \\
Malignant teratoma & $3(4.8)$ & I (local) \\
Fibrosarcoma & $2(3.2)$ & None \\
Malignant mesothelioma & I (I.6) & None \\
\hline
\end{tabular}

underappreciated by clinicians and the tumors are commonly misdiagnosed, even after extensive radiological workup. ${ }^{3}$ The majority of retrorectal tumors are benign; malignant tumors are notoriously difficult to excise for a cure, although they can be slow growing and rarely metastasize until late in their course.

Retrorectal tumors are frequently asymptomatic and are often found incidentally during evaluation for unrelated physical complaints. Furthermore, even patients with symptoms directly referable to a retrorectal tumor may be initially misdiagnosed as having fistulae in ano; pilonidal cysts; perianal abscesses; psychogenic, post-traumatic, or postpartum pain; or proctalgia fugax. In fact, Singer et al found that patients underwent an average of 4.1 surgical procedures before being correctly diagnosed with primary retrorectal pathology. ${ }^{2}$

Retrorectal tumors may produce minimal or vague symptoms for long periods of time. In fact, benign lesions are most often aymptomatic. ${ }^{4}$ Patients may initially complain of constipation, paradoxical diarrhea, or frequent urge to defecate, as the tumor causes compression of the adjacent rectum. Previously, retrorectal tumors in women were occasionally diagnosed during labor as the cause of dystocia. Pain (either pelvic discomfort or sciatic symptoms) is more common with malignant retrorectal tumors. Two large series have demonstrated that pain is present in $86 \%-88 \%$ of patients with malignancy versus $22 \%-39 \%$ of those with benign lesions. ${ }^{2,3}$ Other factors predictive of malignancy include male gender, and age $>60$ years. Chordomas are classically associated with constant dull aching in the low back, pelvis, and buttocks. This pain is typically worsened by sitting and relieved with standing or walking. Patients with anterior sacral meningoceles may complain of headaches 
after straining during defecation or intercourse, and their symptoms may be reproduced with Valsalva. ${ }^{5}$ The diagnosis of retrorectal cyst should be suspected in any patient with a history of repeated attempts at drainage for a fistula in ano or perirectal abscess, especially when no obvious internal rectal opening can be identified or mucoid material (rather than pus) is drained. ${ }^{6}$ Malignant tumors that invade the sacral plexus or nerve roots can lead to bowel and bladder incontinence or urinary retention.

All patients with suspected retrorectal tumors should undergo complete colonoscopy and women should have detailed bimanual pelvic examinations to exclude the much more common conditions of colorectal or ovarian/uterine adenocarcinomas, respectively. The rectal mucosa overlying a retrorectal tumor appears normal. Small lesions may not be detectable on proctoscopy, but larger lesions demonstrate extraluminal compression of the rectum. Traditionally, evaluation of suspected retrorectal tumors begins with plain radiographs of the pelvis. These are typically normal and provide little additional information. The exceptions are malignant tumors that may produce bone destruction of the sacrum, as well as the rare benign bone-based lesions such as aneurismal bone cysts, giant cell tumors, and osteochondromas. ${ }^{6}$ Transrectal ultrasonography appears to have utility in establishing the diagnosis of a retrorectal tumor. Transrectal ultrasonography was found to have a sensitivity of $100 \%$ when combined with rigid proctoscopy. ${ }^{2,4}$ Tomographic imaging, with either computed tomography or MRI, has become the standard for preoperative evaluation of retrorectal tumors. In particular, MRI with an endorectal coil can provide detailed images depicting the relationship of the tumor with sacral nerve roots, the coccyx, and the musculature of the pelvic floor. MRI also appears to be slightly more specific for establishing the histology of the retrorectal tumor. ${ }^{2}$

In appropriate surgical candidates, all retrorectal tumors should be resected, even if asymptomatic. Glasgow and Dietz have previously summarized the reasons for operative extirpation. ${ }^{6}$ For patients who are medically fit for an operation and in whom the lesion seems resectable, treatment of retrorectal lesions is almost always surgical resection. A suitable approach is the key to the successful surgical treatment of the retrorectal tumor. ${ }^{7}$ Three different operative approaches are commonly used in the resection of retrorectal tumors: abdominal, transsacral, and combined abdominosacral. The approach depends on the nature and location of the lesion. Lesions that do not extend below S4 (high lesions) can be resected transabdominally (anterior approach). Lower lesions can be resected transsacrally (posterior approach). If the upper extent of the lesion can be palpated on rectal examination, it is likely to be resectable transsacrally. Larger lesions or those in an intermediate position may require a combined abdominal and sacral operation.

The transsacral approach is preferred for small (diameter $<8 \mathrm{~cm}$ ), benign tumors or for perineal fistulae that do not extend above the level of the fourth sacral element. ${ }^{8}$ This approach enables good access to the caudal component of the tumor, as well as producing excellent results with minimal morbidity. As tumors are resected via a posterior approach the patient takes the Kraske position. A longitudinal or " $\mathrm{S}$ "-shaped incision between the coccyx and the posterior margin of the anus is selected, and the coccyx, as well as the fourth and fifth sacral vertebra, can be resected when necessary to achieve good exposure of the presacral space. In the authors' group, 48 patients $(77.4 \%)$ received a wholly posterior approach for low-lying tumors (ie, under the promontory). This supported the notion that when the superior border of the tumor is palpable and movable during digital examination, the posterior approach should be successful. ${ }^{2}$ However, lesions above the sacral promontory or which are potentially malignant are amenable to the abdominal approach, as it provides direct visualization of the middle sacral artery, and the presacral veins and nerves. ${ }^{1}$

Frequently, the tumor is supplied and drained by the presacral vessels, which must be ligated. When large tumors or infected cysts involve the rectum or the presacral fascia, the inflammation often obscures normal planes and causes significant adhesion to the adjacent tissues. A combined abdominosacral approach is then suggested in these kinds of cases. ${ }^{9}$ The benefits of the combined abdominoperineal approach include improved visualization of structures, such as the ureters and the rectum itself via the anterior incision, as well as enhanced exposure of the nerve roots provided by the posterior approach. In four of the authors' cases, the tumor was firstly exposed in the posterior approach, through which an en bloc tumor resection was difficult to perform. The tumor was then resected through a combined approach. Nevertheless, in another six cases using a direct combined approach, radical tumor resections were performed and smooth recovery was achieved. This indicates that a combined abdominoperineal approach should be chosen when there is evidence for tumors with rectal or adjacent structure or organ (uterus or bladder) involvement.

The development of tumors can cause anatomical variations in the retrorectal space, and is always associated 
with an increased incidence of surgical complications involving the sacral plexus and sympathetic nerves, with an incidence reported to be $36 \%$ by Lev-Chelouche et al. ${ }^{10}$ Bleeding can be caused by impairment of either major vessels or the pre-sacral venous plexus, and is one of the most severe complications of presacral operations. Therefore, great care must be taken to avoid injury to the vessels. Preoperative angiography provides detailed information on vessel distribution and tumor blood supply, and is recommended for tumors of large size or malignant potential. In the authors' experience, resection of presacral tumors should be performed under direct visualization with sharp dissection. Redundant blunt dissection or excessive suction on the sacrum should be avoided to reduce the risk of vessel injury. For large tumors, the sacrum and coccyx can be resected when necessary to achieve a good exposure of tumor and surrounding tissue. For cystic lesions, eliciting the cystic fluid helps to reduce the volume and therefore facilitates resection. For solid tumors, partial resection of the tumor within the membrane helps to differentiate tumor from vicinal tissues such as iliac vessel, rectum, and ureters. An en bloc resection is then performed. In the authors' series, one case with left iliac vessels surrounded by the tumor had left lateral iliac artery splitting and was retrieved by vessel repair. Presacral venous plexus bleeding during dissection of presacral fascia in another two cases was stopped by Xu's hemostasis procedure, which was considered most effective in the present authors' experience. ${ }^{11}$

Frequently, polycystic lesions or malignant tumors in the retrorectal space are densely adherent to the rectum, which increases the incidence of rectal injury during surgical resection. Insertion of one finger into the rectal vault helps to differentiate the rectal muscularis and the surrounding fibers of the levator ani. ${ }^{12}$ Commonly, with one finger inserted between the tumor and rectum, an assistant could help to differentiate the tumor from the rectum to perform an en bloc excision. However, once a cystic lesion is densely adhered to the rectum, blunt dissection should be avoided. The tumor can be partially resected with its content dislodged and the capsule wall that neighbors the rectum preserved on the serous membrane of the rectum. The secretion function of the residual capsule wall is then destroyed with electric coagulation, which minimizes the chance of rectal injury and pelvic cavity infection. In addition, in case of a rectal rupture, either rectal repair or partial rectum resection with anastomosis is feasible for a satisfactory preoperative bowel preparation. However, when the general condition of the patient is poor, rectal ostomy is suggested. The resection of a large presacral tumor is similar to the extended radical resection of a rectal carcinoma, during which inferior abdominal as well as pelvic nerve plexus injury is a common complication, resulting in post-operative urinary functional disturbance of $7 \% \sim 70 \%$ and sexual disturbance of $25 \% \sim 100 \% .{ }^{13}$ According to the authors' experience, the tumor should be prosected and dissected along the presacral fascia and deep rectal fascia under direct visualization. Inferior abdominal nerves can be differentiated when necessary, to reduce the incidence of urinary or sexual function disturbance.

Benign tumors provided a better opportunity for complete resection than did malignant ones. In patients with tumor recurrence, residual cysts comprised the majority of those with benign lesions while local recurrence and metastasis was more common in those with malignancies. Commonly, complete resection is crucial for malignant diseases, as it provides the only chance for prolongation of life. Even partial resection provides opportunity for chemotherapy or radiotherapy, and therefore improves survival. ${ }^{10}$ In the authors' series, three of the seven cases with tumor recurrence received more than three operations. Two of them were pathologically diagnosed multiple dermoid cysts and one was malignant. In the remaining four patients with malignant tumors, the recurrent lesions were unresectable because of metastasis.

\section{Conclusion}

Primary neoplasms of the retrorectal (presacral) space are very rare. These lesions may be congenital or acquired, benign or malignant. They often arise with subtle clinical symptoms, or they may be found incidentally during evaluation for other conditions. Preoperative imaging can provide useful information for operative planning; however, it does not eliminate the need for surgery. Approaches for resection include posterior only and combined abdominoperineal, depending on the characteristics of the lesion. Successful treatment of these enigmatic lesions requires extensive knowledge of pelvic anatomy and expertise in pelvic surgery.

\section{Acknowledgments}

This work was supported by the State Key Basic Research and Development Program of China (973 Program, Grant No. 2009CB521704), National High-tech Research and Development Program of China (863 Program, Grant No. 2006AA02A245), Zhejiang Provincial Science and Technology Project (Grant No. 2009C13021), and Zhejiang Provincial Science Research Foundation for Returned Overseas Chinese Scholars (No: J20100113). 


\section{Disclosure}

The authors report no conflicts of interest in this work.

\section{References}

1. Wolpert A, Beer-Gabel M, Lifschitz O, Zbar AP. The management of presacral masses in the adult. Tech Coloproctol. 2002;6(1):43-49.

2. Singer MA, Cintron JR, Martz JE, Schoetz DJ, Abcarian H. Retrorectal cyst: a rare tumor frequently misdiagnosed. J Am Coll Surg. 2003; 196(6):880-886.

3. Jao SW, Beart RW Jr, Spencer RJ, Reiman HM, Ilstrup DM. Retrorectal tumors. Mayo Clinic experience, 1960-1979. Dis Colon Rectum. 1985; 28(9):644-652.

4. Glasgow SC, Birnbaum EH, Lowney JK, et al. Retrorectal tumors: a diagnostic and therapeutic challenge. Dis Colon Rectum. 2005; 48(8):1581-1587.

5. Oren M, Lorber B, Lee SH, Truex RC Jr, Gennaro AR. Anterior sacral meningocele: report of five cases and review of the literature. Dis Colon Rectum. 1977;20(6):492-505.

6. Glasgow SC, Dietz DW. Retrorectal tumors. Clin Colon Rectal Surg. 2006;19(2):61-68.
7. Woodfield JC, Chalmers AG, Phillips N, Sagar PM. Algorithms for the surgical management of retrorectal tumours. Br J Surg. 2008; 95(2):214-221.

8. Abel ME, Nelson R, Prasad ML, Pearl RK, Orsay CP, Abcarian H. Parasacrococcygeal approach for the resection of retrorectal developmental cysts. Dis Colon Rectum. 1985;28(11):855-858.

9. Böhm B, Milsom JW, Fazio VW, Lavery IC, Church JM, Oakley JR. Our approach to the management of congenital presacral tumors in adults. Int J Colorectal Dis. 1993;8(3):134-138.

10. Lev-Chelouche D, Gutman M, Goldman G, et al. Presacral tumors: a practical classification and treatment of a unique and heterogeneous group of diseases. Surgery. 2003;133(5):473-478.

11. Xu J, Lin J. Control of presacral hemorrhage with electrocautery through a muscle fragment pressed on the bleeding vein. $J$ Am Coll Surg. 1994;179(3):351-352.

12. Miles RM, Stewart GS Jr. Sacrococcygeal teratomas in adult. Ann Surg. 1974;179(5):676-683.

13. Mancini R, Cosimelli M, Filippini A, et al. Nerve-sparing surgery in rectal cancer: feasibility and functional results. J Exp Clin Cancer Res. 2000;19(1):35-40.
OncoTargets and Therapy

\section{Publish your work in this journal}

OncoTargets and Therapy is an international, peer-reviewed, open access journal focusing on the pathological basis of all cancers, potential targets for therapy and treatment protocols employed to improve the management of cancer patients. The journal also focuses on the impact of management programs and new therapeutic agents and protocols on

\section{Dovepress}

patient perspectives such as quality of life, adherence and satisfaction The manuscript management system is completely online and includes a very quick and fair peer-review system, which is all easy to use. Visit http://www.dovepress.com/testimonials.php to read real quotes from published authors. 